\title{
Rotated domain network in graphene on cubic-SiC(001)
}

\author{
Alexander N. Chaika, ${ }^{* 1,2}$ Olga V. Molodtsova, ${ }^{3}$ Alexei A. Zakharov, ${ }^{4}$ \\ Dmitry Marchenko, ${ }^{5}$ Jaime Sánchez-Barriga, ${ }^{5}$ Andrei Varykhalov, ${ }^{5}$ \\ Sergey V. Babenkov, ${ }^{5}$ Marc Portail, ${ }^{6}$ Marcin Zielinski, ${ }^{7}$ Barry E. Murphy, ${ }^{2}$ \\ Sergey A. Krasnikov, ${ }^{2}$ Olaf Lübben, ${ }^{2}$ Igor V. Shvets, ${ }^{2}$ and Victor Y. Aristov ${ }^{1,3}$ \\ ${ }^{1}$ Institute of Solid State Physics RAS, Chernogolovka, Moscow district 142432, Russian \\ Federation \\ ${ }^{2}$ Centre for Research on Adaptive Nanostructures and Nanodevices (CRANN), School of Physics, \\ Trinity College Dublin, Dublin 2, Ireland \\ ${ }^{3}$ HASYLAB at DESY, D-22607 Hamburg, Germany \\ ${ }^{4}$ MAX-lab, Lund University, Box 118, 22100 Lund, Sweden \\ ${ }^{5}$ Helmholtz-Zentrum Berlin für Materialien und Energie, D-12489 Berlin, Germany \\ ${ }^{6}$ CNRS-CRHEA, 06560 Valbonne, France \\ ${ }^{7}$ NOVASiC, BP267-F73375 Le Bourget du Lac Cedex, France \\ *e-mail: chaika@issp.ac.ru, chaikaa@tcd.ie
}

\begin{abstract}
The atomic structure of the cubic-SiC(001) surface during ultra high vacuum graphene synthesis has been studied using scanning tunneling microscopy (STM) and low energy electron diffraction. Atomically resolved STM studies prove the synthesis of a uniform, millimeter-scale graphene overlayer consisting of nanodomains rotated by $\pm 13.5^{\circ}$ relative to the $\langle 110\rangle$-directed boundaries. The preferential directions of the domain boundaries coincide with the directions of carbon atomic chains on the $\mathrm{SiC}(001)-\mathrm{c}(2 \times 2)$ reconstruction, fabricated prior to graphene synthesis. The presented data show the correlation between the atomic structures of the $\operatorname{SiC}(001)-\mathrm{c}(2 \times 2)$ surface and graphene/SiC(001) rotated domain network and pave the way for optimizing large-area graphene synthesis on low-cost cubic-SiC $(001) / \mathrm{Si}(001)$ wafers.
\end{abstract}

\section{Introduction}

Its unique electronic properties [1-3] make graphene a very appealing material for future applications. However, to be considered as a potential candidate to replace silicon in electronics, graphene should be controllably grown on large-area insulating substrates compatible with existing lithographic technology. Synthesis on hexagonal silicon-carbide $(\alpha-\mathrm{SiC})$ substrates [4-12] is one of the most promising methods for graphene fabrication on insulating substrates. It is known that even uniform multilayers of graphene on carbon-terminated $\mathrm{SiC}(000-1)$ substrates possess the physical properties and electronic spectra of a free-standing graphene monolayer [8,9]. However, the graphene synthesized on high-cost wafers cut from bulk $\alpha$-SiC single crystals cannot be considered as a viable candidate for industrial mass production. Recently it has been shown that synthesis on low-cost cubic-SiC wafers [13-18] represent a realistic method for massproducing graphene layers suitable for electronic applications. In particular, $\mathrm{SiC}(001)$ thin films grown on $\mathrm{Si}(001)$ wafers are fully compatible with existing technologies. However, the first papers on
graphene/SiC(001) [13-16] brought no information about the graphene overlayer's continuity on the millimeter-scale and provided contradictory information about the atomic and electronic structure of graphene on $\mathrm{SiC}(001)$. In our recent studies [18] it has been demonstrated that the uniform graphene overlayer synthesized on $\mathrm{SiC}(001)$ consists of only a few monolayers with the physical properties of quasifreestanding graphene. However, contrary to the previous results suggesting micrometer-scale graphene flakes on $\mathrm{SiC}(001)$ [14], our scanning tunneling microscopy (STM), low energy electron diffraction (LEED) and angle resolved photoelectron spectroscopy (ARPES) studies [18] have demonstrated the formation of nanometer-sized graphene domains with four preferential orientations. The formation of rotated domains and grain boundaries can modify the electronic properties of graphene and lead to the opening of a transport gap [19], which is crucial for technological applications. Therefore, detailed atomic-scale information about graphene growth on the $\mathrm{SiC}(001)$ surface is important for understanding the physical properties of the graphene domain network and further improvement of the graphene/SiC(001) synthesis procedure. Only 

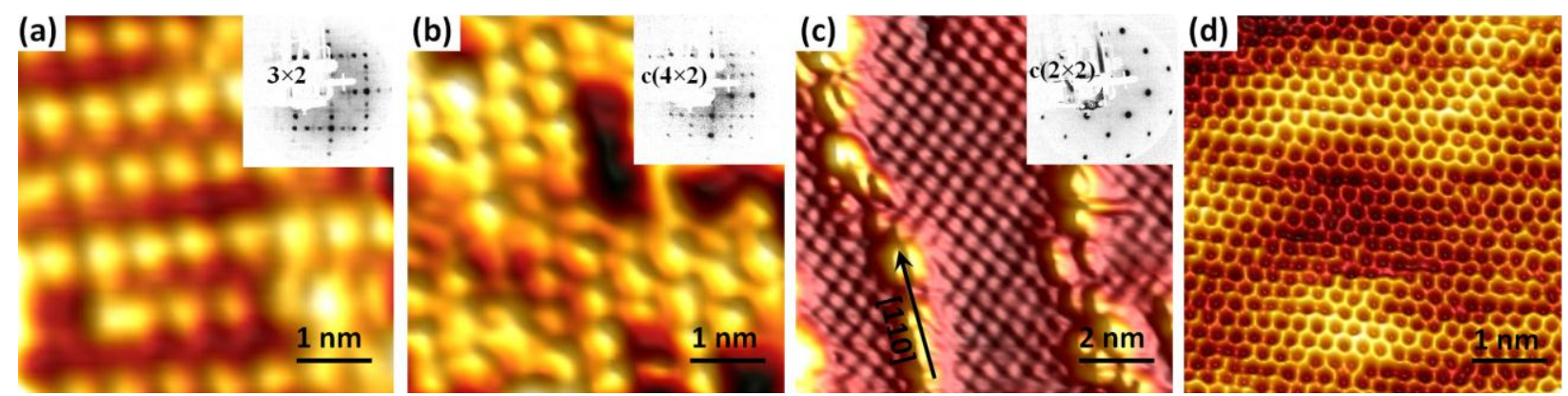

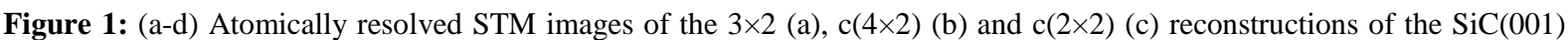
surface and graphene/SiC(001) system (d). Inset in panels (a-c) are typical two-domain LEED patterns of the corresponding surface atomic structures. The images were measured at $U=-2.3 \mathrm{~V}$ and $I=70 \mathrm{pA}(\mathrm{a}), U=-2.5 \mathrm{~V}$ and $I=80 \mathrm{pA}$ (b), $U=-3.0 \mathrm{~V}$ and $I=70 \mathrm{pA}(\mathrm{c}), U=22 \mathrm{mV}$ and $I=70 \mathrm{pA}(\mathrm{d})$. The LEED patterns were taken at different electron beam energies to obtain the best patterns.

step-by-step STM studies can disclose precise information on the surface transformations on the atomic level, test the correlation between substrate defects and graphene layer quality, and, most importantly, inspire new ideas on possible improvements to graphene synthesis on this technologically appealing substrate. High-resolution scanning probe microscopy studies can also provide information about the integrity of the graphene overlayer on the nanometer scale. This is not possible by spectroscopic techniques which average information over micrometer-scale surface areas. Here we present the results of comprehensive STM and LEED studies of the atomic structure of the $\mathrm{SiC}(001)$ surface carried out during all stages of ultra-high vacuum (UHV) synthesis of graphene on $\mathrm{SiC}(001)$. Since different $\mathrm{SiC}(001)$ reconstructions were studied previously [20-26], in our STM experiments we focus not on the highest quality $\mathrm{SiC}(001)$ atomic structures but on the most typical reconstructions formed prior to graphene synthesis. To directly compare possible defects on the SiC surface and the graphene overlayer we have performed successive STM experiments on the same samples at each stage of the treatments necessary for graphene synthesis. STM studies were conducted on numerous spatially separated surface regions to obtain detailed information on the uniformity of the synthesized graphene on the millimeter-scale and its correlation with the atomic structure of $\mathrm{SiC}(001)$.

\section{Experimental}

Graphene layers were fabricated on cubic-SiC $(001)$ films grown on on-axis $\mathrm{Si}(001)$ wafers using $\mathrm{Si}$-atom sublimation followed by surface layer graphitization at high temperatures. The atomic structure of the $\mathrm{SiC}(001)$ surface during graphene synthesis was studied in-situ in a RIBER LAS-3000 spectrometer equipped with a room temperature (RT) GPI-300 STM. Chemically etched [001]- and [111]-oriented single-crystalline tungsten tips sharpened in UHV by electron beam heating and ion sputtering [27,28] were used for STM experiments. The base pressure in the analytical chamber was in the range of $4-6 \times 10^{-}$ ${ }^{11}$ mbar. It did not exceed $2 \times 10^{-10}$ mbar during the direct current heating of the $3 \times 8 \times 0.5 \mathrm{~mm}^{3}$
$\mathrm{SiC}(001) / \mathrm{Si}(001)$ wafers and rapidly recovered after the sample treatments. To obtain a lower pressure in the UHV chamber during graphene synthesis, $\mathrm{SiC}(001) / \mathrm{Si}(001)$ wafers were thoroughly outgassed at $600-700^{\circ} \mathrm{C}$ for several hours to reach a pressure in the $10^{-11}$ mbar region. The samples were then flashed at $\sim 1100^{\circ} \mathrm{C}$ to remove contaminants. After the flash heating, several monolayers (MLs) of silicon atoms were deposited onto the carbon-rich $\operatorname{SiC}(001)-1 \times 1$ surface at a deposition rate of $\sim 1 \mathrm{ML} / \mathrm{min}$. Next, the $\mathrm{SiC}(001)$ surface was annealed at gradually increasing temperatures, giving rise to $\mathrm{Si}$ atom sublimation and the fabrication of different surface reconstructions. STM experiments were conducted after cooling the samples to RT. STM data were analyzed using WSXM software [29]; either raw data or STM images smoothed by a $3 \times 3$ matrix are presented.

\section{Results and discussion}

The first step towards graphene sythesis on $\mathrm{SiC}(001)$ is sustained annealing to fabricate a uniform, Si-rich $\mathrm{SiC}(001)-3 \times 2$ reconstructed surface with large terraces [22]. Long-term annealing at temperatures of $700-800^{\circ} \mathrm{C}$ was applied. Increasing the annealing temperature from $800^{\circ} \mathrm{C}$ to $1300^{\circ} \mathrm{C}$ causes the surface to undergo consecutive Si-terminated $5 \times 2, \mathrm{c}(4 \times 2)$, $2 \times 1$, and C-terminated $c(2 \times 2)$ reconstructions in accordance with the results of Refs. [20-26]. Figures 1a-c show atomically resolved STM images of the $3 \times 2, \mathrm{c}(4 \times 2)$ and $\mathrm{c}(2 \times 2)$ reconstructions formed on our $\mathrm{SiC}(001)$ sample at the different stages of graphene synthesis. Note that images of the $c(4 \times 2)$ reconstruction usually revealed missing $\mathrm{Si}$ atoms in the surface layer (Fig. 1b), while the $c(2 \times 2)$ structure was typically decorated by excessive carbon atoms and atomic chains elongated in the [110] direction (Fig. 1c). The absence of impurities on the $\mathrm{SiC}(001)$ surface is confirmed by the fabrication of the $c(4 \times 2)$ reconstruction (Fig. 1b) which is very sensitive to contamination. It usually transforms into the $2 \times 1$ phase after several hours due to exposure to background hydrogen in UHV [24]. The excess of carbon atoms observed on the $\operatorname{SiC}(001)-c(2 \times 2)$ as bright protrusions on terraces (Figs. 1c and 2a), can help to transform the $\mathrm{c}(2 \times 2)$ reconstruction into the 

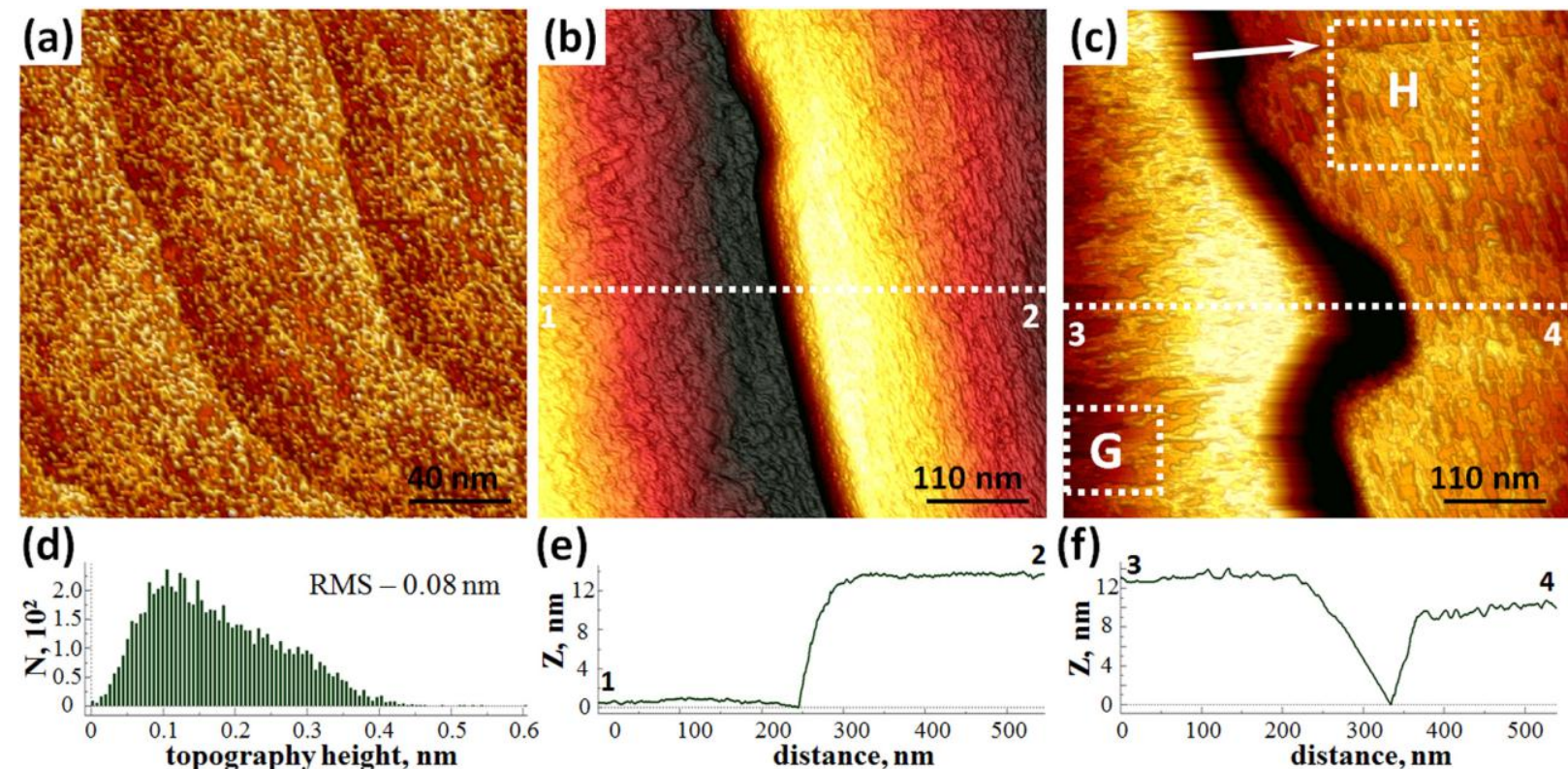

2 (f)
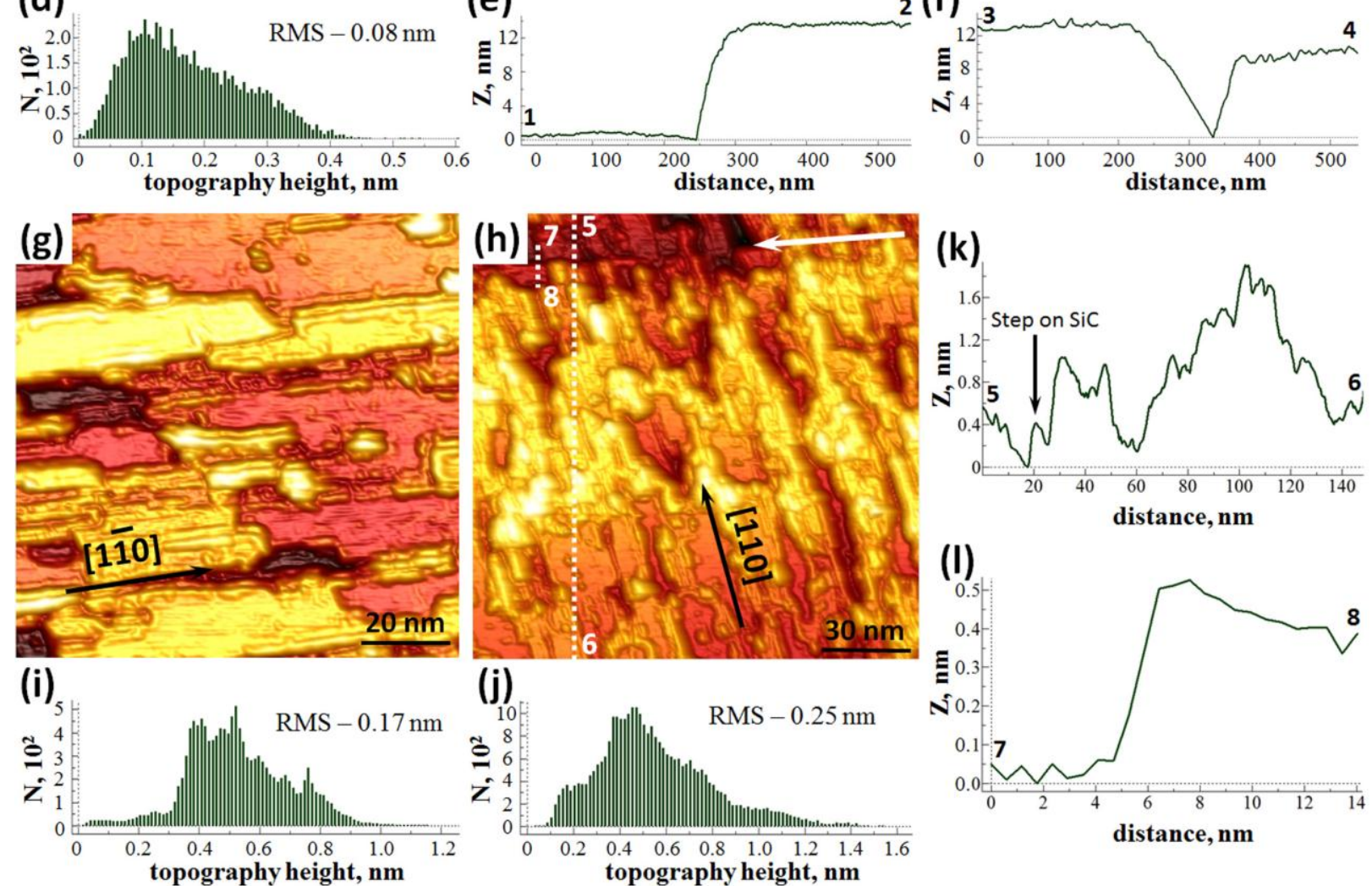

Figure 2: Large area STM images of the $\mathrm{SiC}(001)-\mathrm{c}(2 \times 2)$ (a) and graphene/SiC(001) (b, c, g, and h). The images in panels (b) and (c) illustrate that the graphene overlayer is not broken by the typical defects of the $\mathrm{SiC}(001)$ substrate. The images in panels $(\mathrm{g})$ and $(\mathrm{h})$ emphasize the horizontal $(\mathrm{g})$ and vertical $(\mathrm{h})$ nanoribbons observed on the left and right side of the APD boundary $(\mathrm{c})$, respectively. The images in panels $(\mathrm{g})$ and $(\mathrm{h})$ were measured from the surface areas labeled $\mathrm{G}$ and $\mathrm{H}$ in panel (c). The STM images were measured at $U=-3.0 \mathrm{~V}$ and $I=60 \mathrm{pA}(\mathrm{a}), U=-1.0 \mathrm{~V}$ and $I=60 \mathrm{pA}(\mathrm{b}), U=-0.8 \mathrm{~V}$ and $I=50$ pA (c), $U=-0.8 \mathrm{~V}$ and $I=60 \mathrm{pA}(\mathrm{g}), U=-0.7 \mathrm{~V}$ and $I=70 \mathrm{pA}(\mathrm{h})$. The white arrows in panels (c) and (h) indicate a single atomic step on the SiC substrate. $(\mathrm{d}, \mathrm{i}, \mathrm{j})$ Roughness analysis of the STM images in panels $(\mathrm{a}, \mathrm{g}, \mathrm{h})$. The histograms were calculated from surface areas of the same size $\left(100 \times 100 \mathrm{~nm}^{2}\right)$ for direct comparison of the $\mathrm{SiC}(001)$ roughness before and after graphene synthesis. (e, f, k, and l) Cross-sections (1-2), (3-4), (5-6) and (7-8) taken from the images in panels (b), (c) and (h).

more densely packed honeycomb lattice (Fig. 1d). Nevertheless, the single atomic steps are well resolved on large area STM images (Fig. 2a). The root mean square roughness (RMS) of micrometerscale $\left(1 \times 1 \mu \mathrm{m}^{2}\right)$ images of the $\mathrm{SiC}(001)-\mathrm{c}(2 \times 2)$ surface did not usually exceed $1.5 \AA$. As an example, the roughness analysis for a smaller surface area is shown in Fig. 2d. Since the apparent height in STM images can strongly depend on the tip and surface electronic structure as well as the tunneling parameters, we have analyzed the heights of monatomic steps on large-area images of different
$\mathrm{SiC}(001)$ reconstructions and the graphene/ $\mathrm{SiC}(001)$ system. We have found that at bias voltages greater than $\pm 0.8 \mathrm{~V}$, the heights of monatomic steps are in good agreement with the well-known cubic-SiC lattice parameters. This is illustrated by crosssections (Figs. 2k and 2l) from the STM image measured near the monatomic step on graphene/SiC(001) (Fig. 2h). Therefore, we can assume that the RMS values shown in Fig. 2 correspond to the actual roughness of the surfaces.

To minimize the depletion of $\mathrm{Si}$ atoms from the $\mathrm{SiC}(001)$ surface and exclude the growth of 

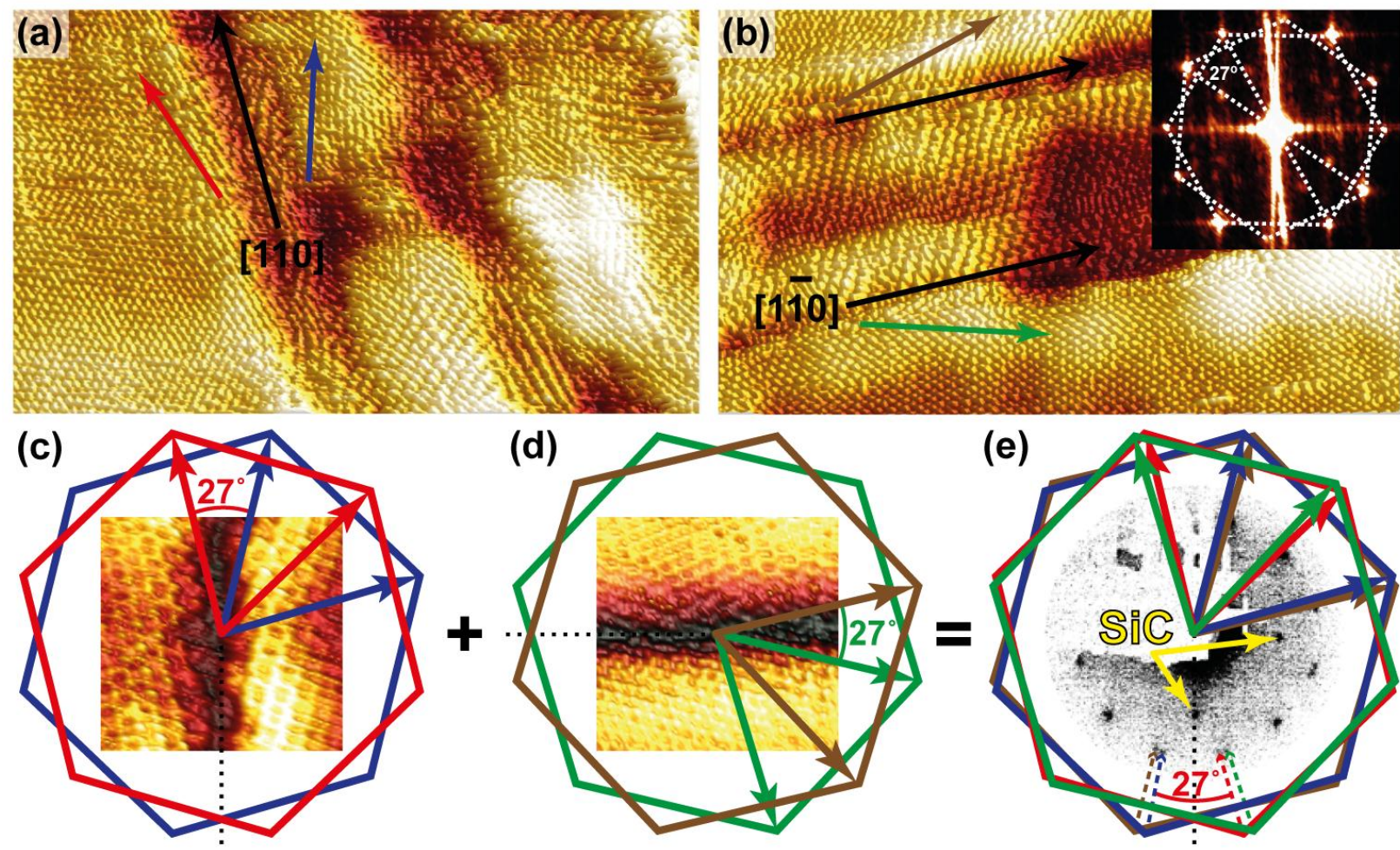

Figure 3: (a,b) $18 \times 11 \mathrm{~nm}^{2}$ atomically resolved STM images of the vertical (a) and horizontal (b) nanoribbons. The images were taken at $U=10 \mathrm{mV}$ and $I=60 \mathrm{pA}$. Inset in panel (b) shows an FFT pattern with two $27^{\circ}$-rotated systems of spots. (c-e) Models explaining the origin of the 24 diffraction spots in the LEED patterns of graphene/SiC $(001)$. Insets in panels (c) and (d) are STM images of the $\langle 110\rangle$-directed domain boundaries. The four different coloured hexagons, red, blue, green and brown represent the four domain orientations, indicated by similarly-coloured arrows in (a) and (b). Inset in panel (e) shows a LEED pattern taken from graphene $/ \mathrm{SiC}(001)$ at $E_{p}=65 \mathrm{eV}$, demonstrating $1 \times 1$ substrate spots (highlighted by yellow arrows) along with 12 double-split graphene monolayer spots, indicated by one dotted arrow for each orientation

multilayer graphite-like structures, only short flashes (20-30 seconds) at temperatures between 1300 $1350^{\circ} \mathrm{C}$ were applied to convert the $\mathrm{c}(2 \times 2)$ reconstruction (Fig. 1c) into the honeycomb graphene lattice (Fig. 1d). Usually after several flashes, the $\mathrm{SiC}(001)-\mathrm{c}(2 \times 2)$ structure was transformed into a uniform domain-like graphene overlayer as our STM (Figs. 2, 3a and 3b) and LEED data (Fig. 3e) confirm. The short flash method used in this work is similar to that used for the growth of graphene on $\alpha-\mathrm{SiC}$ $[10,11,30,31]$.

After graphene synthesis we did not observe any bare silicon carbide regions on the graphene/SiC $(001)$ samples. As the large area STM images in Figs. $2 \mathrm{~g}$ and $2 \mathrm{~h}$ illustrate, the surface layer consists of nanodomains connected through domain boundaries. Comparing STM data obtained before (Fig. 1c) and after (Figs. $2 \mathrm{~g}$ and $2 \mathrm{~h}$ ) graphene synthesis, it can be concluded that the nanodomain boundaries are preferentially aligned along the $\langle 110\rangle$ directions of the $\mathrm{SiC}$ crystal lattice as indicated in Figs. $2 \mathrm{~g}$ and $2 \mathrm{~h}$. The domains are typically elongated in either the [110] or [1-10] direction, have widths in the range of 5 to $30 \mathrm{~nm}$ and lengths varying from 20 to $200 \mathrm{~nm}$. The average width of these nanodomains is approximately $10 \mathrm{~nm}$, although wider nanoribbons are frequently observed.

The coincidence of the directions of carbon atomic chains and graphene domain boundaries shows that the top graphene layer, which is formed first during the "bottom-up" graphene growth, follows the nanometer-scale morphology of the $\mathrm{SiC}(001)-\mathrm{c}(2 \times 2)$ surface. This is similar to graphene growth on $\alpha$-SiC[4-12,30,31]. However, because of the different symmetries of the cubic $\operatorname{SiC}(001)$ and honeycomb graphene lattices, they cannot match each other as closely as graphene and hexagonal SiC. This reduces the interaction between the graphene layer and the $\mathrm{SiC}(001)$ substrate and leads to the absence of a reactive buffer layer on $\mathrm{SiC}(001)[13,18]$, unlike on $\alpha$-SiC $[4-12,30,31]$. It can be supposed that the diamond-like carbon chains may be an obstacle for the growth of larger (micrometer-scale) graphene domains on $\mathrm{SiC}(001)$. Therefore, controlling the density and orientation of the chains on $\mathrm{SiC}(001)$ $c(2 \times 2)$ could allow the average size of the graphene domains and their orientation to be tuned.

Individual domains possess a rippled morphology, which leads to a root mean square roughness of the large area STM images ranging from $1.5 \AA$ to $4.0 \AA$ (e.g., see Figs. $2 \mathrm{i}$ and $2 \mathrm{j}$, which demonstrate an RMS of $1.7 \AA$ and $2.5 \AA$ for $100 \times 100$ $\mathrm{nm}^{2}$ surface areas, respectively). Note that the roughness calculated from images of the same size is approximately a factor of two larger for graphene/SiC $(001)$ than for $\operatorname{SiC}(001)-c(2 \times 2)$. The roughness of $1 \times 1 \quad \mu \mathrm{m}^{2}$ STM images of graphene/ $\mathrm{SiC}(001)$ was usually between 2.5 and 4.5 $\AA$. These values are typical of free-standing, singlelayer graphene $[32,33]$ and substantially exceed the 

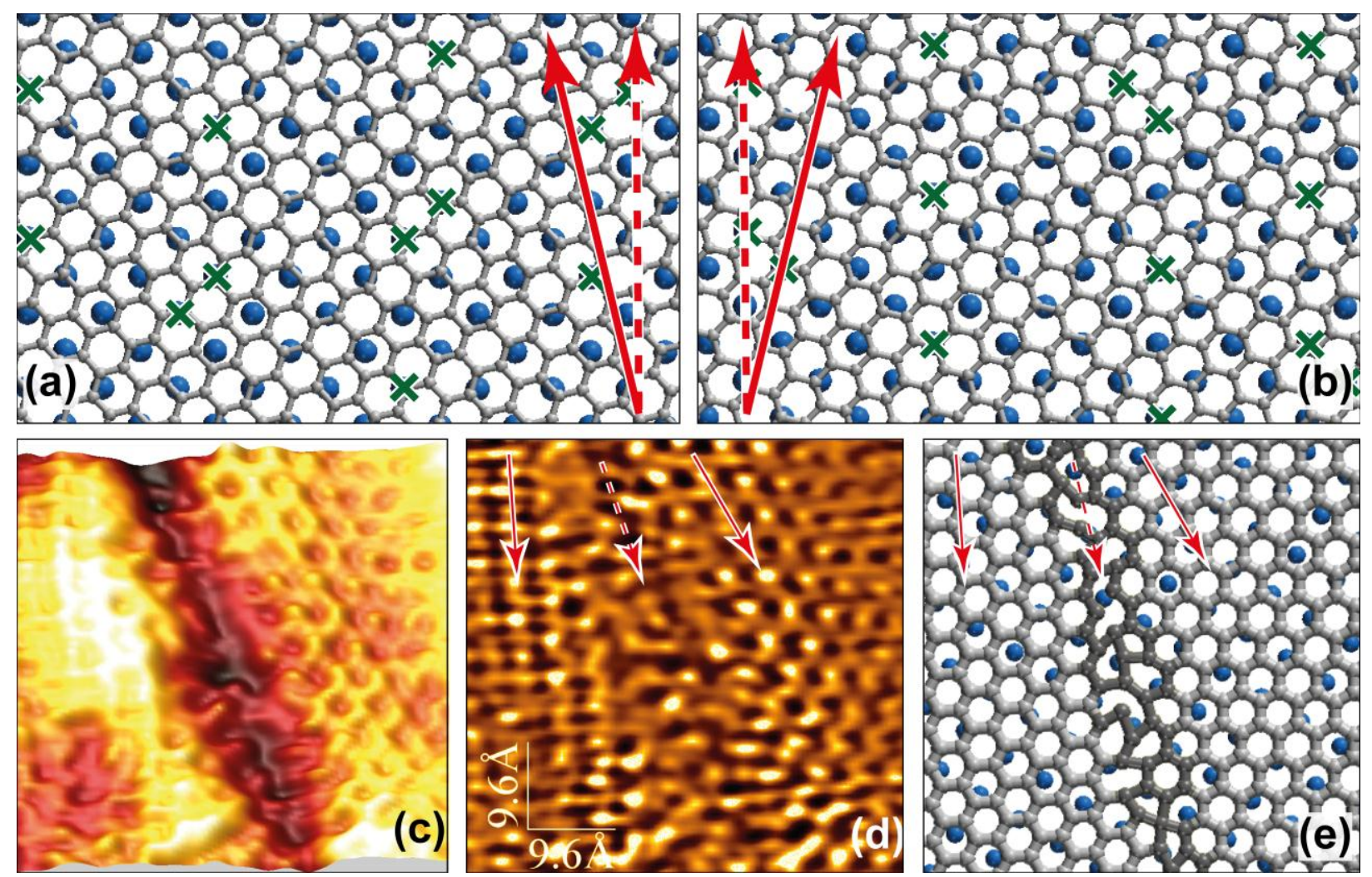

Figure 4: $(\mathrm{a}, \mathrm{b})$ Schematic models representing two different domains. The zigzag direction of the graphene lattice is rotated by $13.5^{\circ}$ counter-clockwise (a) and clockwise (b) with respect to the [110] direction of the underlying C-terminated $\mathrm{SiC}(001)$ surface. The $\mathrm{SiC}[110]$ direction is indicated by the dashed arrow, and the graphene zigzag direction by the solid arrow. Coinciding lattice points between the graphene layer (grey spheres) and the $\mathrm{SiC}$ carbon atoms (blue spheres) are emphasised by green crosses. (c) 3D image of a $4.8 \mathrm{~nm} \times 4.8 \mathrm{~nm}$ area of the graphene surface showing a typical boundary between two rotated graphene domains, showing the bending of the layer where the two domains meet. (d) 2D STM image of the same area as in (c). This image has been smoothed by a Laplacian function to highlight the disordered atomic structure of the domain boundary [29]. (e) Schematic model of a similar boundary, illustrating the incommensurate nature of the two domains. The darker grey atoms indicate the zone where they cannot adopt the regular graphene honeycomb structure.

\section{$\mathrm{SiC}(001)$ roughness prior to graphene fabrication.}

The orientation of the graphene domains is linked to the atomic structure of the $\mathrm{SiC}(001)-\mathrm{c}(2 \times 2)$ surface decorated by $\langle 110\rangle$-directed carbon atomic chains (Fig. 1c). Nanodomains elongated in the [110] (Fig. 2g) and [110] (Fig. 2h) directions are resolved on the left and right side of the anti-phase domain (APD) boundary shown in Fig. 2c. It is noted that imaging the surface area near the boundary was stable even at a sample bias voltage of $-0.8 \mathrm{~V}$, which lies within the band gap of $\mathrm{SiC}(2.3 \mathrm{eV})$. Although the resolution is limited near the APD boundary and actual topography of the boundary can not be seen because of tip effects, the absence of a jump-tocontact at such a small bias voltage confirms the absence of bare $\mathrm{SiC}(001)$ regions at the edges of the APD boundary defect. Additional proof of the continuity of the graphene overlayer at the APD boundaries was obtained during LEEM experiments. Reflectivity measurements from small $(\sim 500 \mathrm{~nm}$ in diameter) surface areas inside the domains and at the APD boundaries reveal the same spectra for each, with three distinct minima [18] proving a uniform graphene thickness throughout the sample. A surface region containing another defect typical of the $\mathrm{SiC}$ substrate (multiatomic step) is shown in Fig. 2 b. Again, imaging is stable throughout the whole scanned area at a bias voltage of $-1.0 \mathrm{~V}$. This proves that the graphene overlayer is continuous near the step edge and uniformly covers the $\mathrm{SiC}$ substrate. Thus, our STM data show that, despite the presence of APD boundaries and multiatomic step defects typical of cubic-SiC, the continuity of the graphene overlayer synthesised on $\mathrm{SiC}(001)$ is most probably unbroken. This is very important for the reproducible synthesis of large-area, uniform graphene layers. However, the APD defects lead to the rotation of the nanodomains by $90^{\circ}$ as Figs. $2 \mathrm{~g}$ and $2 \mathrm{~h}$ illustrate. The well-resolved monatomic step on the $\mathrm{SiC}$ substrate under the graphene domain network (Fig. 2h), emphasized by the cross-sections in Figs. 2k and 2l, confirms that the graphene coverage does not exceed a few monolayers.

Atomically resolved images in Figs. 3a and 3b, which show STM images of domains elongated in the [110] and [1-10] directions, respectively, reveal the atomic structure of the graphene domain network on $\mathrm{SiC}(001)$. The 2-dimensional fast Fourier transform (FFT) patterns of the STM images (see inset in Fig. 3b) consist of two systems of spots (indicated by hexagons), which are related to two graphene domains rotated by $27^{\circ}$ relative to each other. It is noted that the graphene domains' lattices are preferentially rotated by $\pm 13.5^{\circ}$ from domain 
boundaries along the [110] and [1-10] directions. These two families of $27^{\circ}$-rotated domains are themselves rotated by $90^{\circ}$ relative to one another and produce two systems of 12 non-equidistant diffraction spots (Fig. 3c and 3d). Together these two rotated diffraction patterns produce the LEED pattern of graphene/SiC(001) with 12 double-split spots, as Fig. 3e illustrates. These rotated domain families are resolved as horizontal and vertical nanoribbons in STM images, as shown in Figs. $2 \mathrm{~g}$ and $2 \mathrm{~h}$.

Figs. $4 \mathrm{a}$ and $4 \mathrm{~b}$ show a schematic model of the graphene domains on $\mathrm{SiC}(001)$ rotated by $\pm 13.5^{\circ}$ from the $\langle 110\rangle$-directed boundaries. One can see that the atomic lattices of the rotated graphene domains and the substrate coincide only in some sites for this misalignment angle, but additional points of coincidence can appear due to the rippling and random distortions of the carbon bond lengths [18]. The graphene and $\mathrm{SiC}(001)$ lattices ideally coincide only at some sites, which leads to a weak interaction between the substrate and overlayer and the absence of a buffer layer in the graphene/SiC(001) system $[13,18]$. One can see from Fig. 4 that the points of coincidence of the substrate and graphene lattices are aligned along one of the $\langle 110\rangle$ directions of the $\mathrm{SiC}$ lattice, corresponding to the direction of atomic chains on the $\mathrm{SiC}(001)-\mathrm{c}(2 \times 2)$ reconstruction. Most probably, these diamond-like chains determine the orientation of the domain boundaries in the topmost graphene layer. Because of the delicate balance between the graphene-SiC(001) interaction and domain boundary energies, the $13.5^{\circ}$ misalignment angle can be more favorable than other rotation angles which would give a better coincidence between the graphene honeycomb and $\mathrm{SiC}(001)$ square lattices. High resolution images (Figs. 4c and 4d) show two typical features related to the nanodomain boundaries in graphene/SiC(001). The layer near the boundary is bent leading to an additional roughness on the order of several Ångströms (Fig. 4c). This roughness can be caused by stress due to the conjunction of neighbouring domains but it can also be observed near the domain edges in freestanding single layer graphene [33]. Fig. $4 \mathrm{~d}$ also reveals an irregular atomic structure of the boundary, which is in good agreement with the domain boundary schematic shown in Fig. 4e.

Since the graphene growth on the $\mathrm{SiC}(001)$ follows a "bottom-up" scenario [12], the growth conditions can be different for the graphene layers which grow under the topmost domain-like graphene layer. The surface's depletion of Si atoms, leading to carbon atomic chain formation on the $\mathrm{SiC}(001)$, can be suppressed after the synthesis of the first graphene layer. Therefore, the lower lying graphene layers can be rotated relative to the topmost layer, similar to multilayer graphene on $\alpha-\mathrm{SiC}[8,9]$. This can reduce the interaction between the graphene layers and preserve the free-standing monolayer-like properties of few layer graphene on $\mathrm{SiC}(001)$ [18].

STM images taken from surface regions far from the domain boundaries reveal defect-free honeycomb (Fig. 1d) or hexagonal (Fig. 5a) lattices distorted by

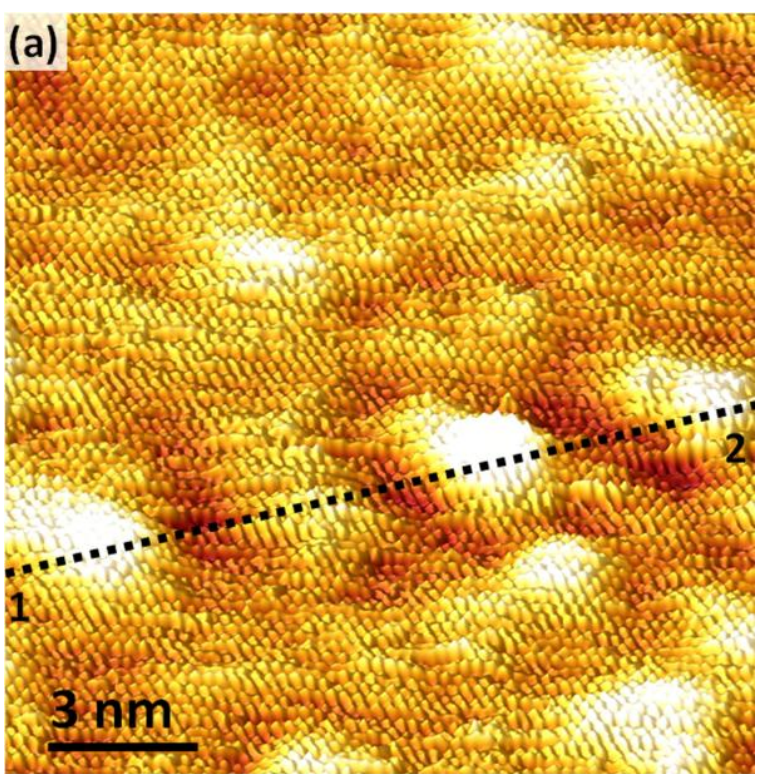

(b)

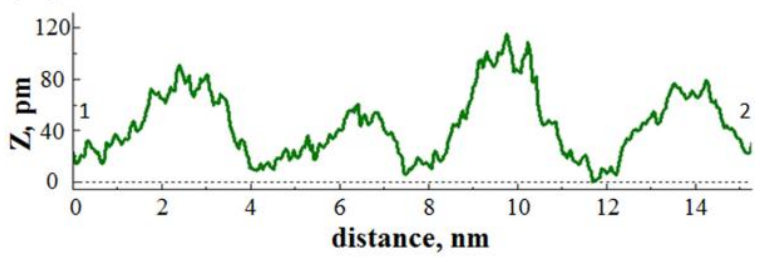

Figure 5: (a) $15 \times 15 \mathrm{~nm}^{2} \mathrm{STM}$ image of a domain region illustrating atomic-scale rippling typical of a freestanding graphene layer. The image was measured at $U=0.1 \mathrm{~V}$ and $I=60 \mathrm{pA}$. (b) Cross-section (1-2) from the image in panel (b) demonstrating that the widths and heights of the ripples in graphene/SiC $(001)$ are in full agreement with theoretical calculations [33].

atomic-scale rippling. As shown in Figs. 5a and 5b, the dimensions of the graphene ripples are on the order of several nanometers laterally and $1 \AA$ vertically, coinciding with such values calculated for a free-standing monolayer [33]. Such values were also observed in STM experiments on an exfoliated graphene monolayer supported by a $\mathrm{SiO}_{2} / \mathrm{Si}$ substrate [34]. It has been demonstrated recently that the apparent height of these ripples can be enhanced in STM experiments on free-standing graphene because of strong tip-sample interactions and the high flexibility of the graphene monolayer $[34,35]$. This is shown in Figs. $6 a$ and $6 b$, where a substantial change in the surface topography is detected with only a minor increase in the tunneling current, similar to effects observed on graphene/ $/ \mathrm{SiO}_{2}$ [36]. The roughness of the surface layer can be modified by the tip-surface interaction after an increase in the tunneling current of only $33 \%$ (which corresponds to a change in the tip-sample distance of just several picometers), further supporting the quasi-freestanding character of graphene on $\mathrm{SiC}(001)$. The images in Figs. $6 \mathrm{a}$ and $6 \mathrm{~b}$ also show that some surface regions (indicated by an arrow) which look like boundaries within the domain network at some tunneling parameters, can in fact be related to a bent single layer.

The STM data show that images with either 

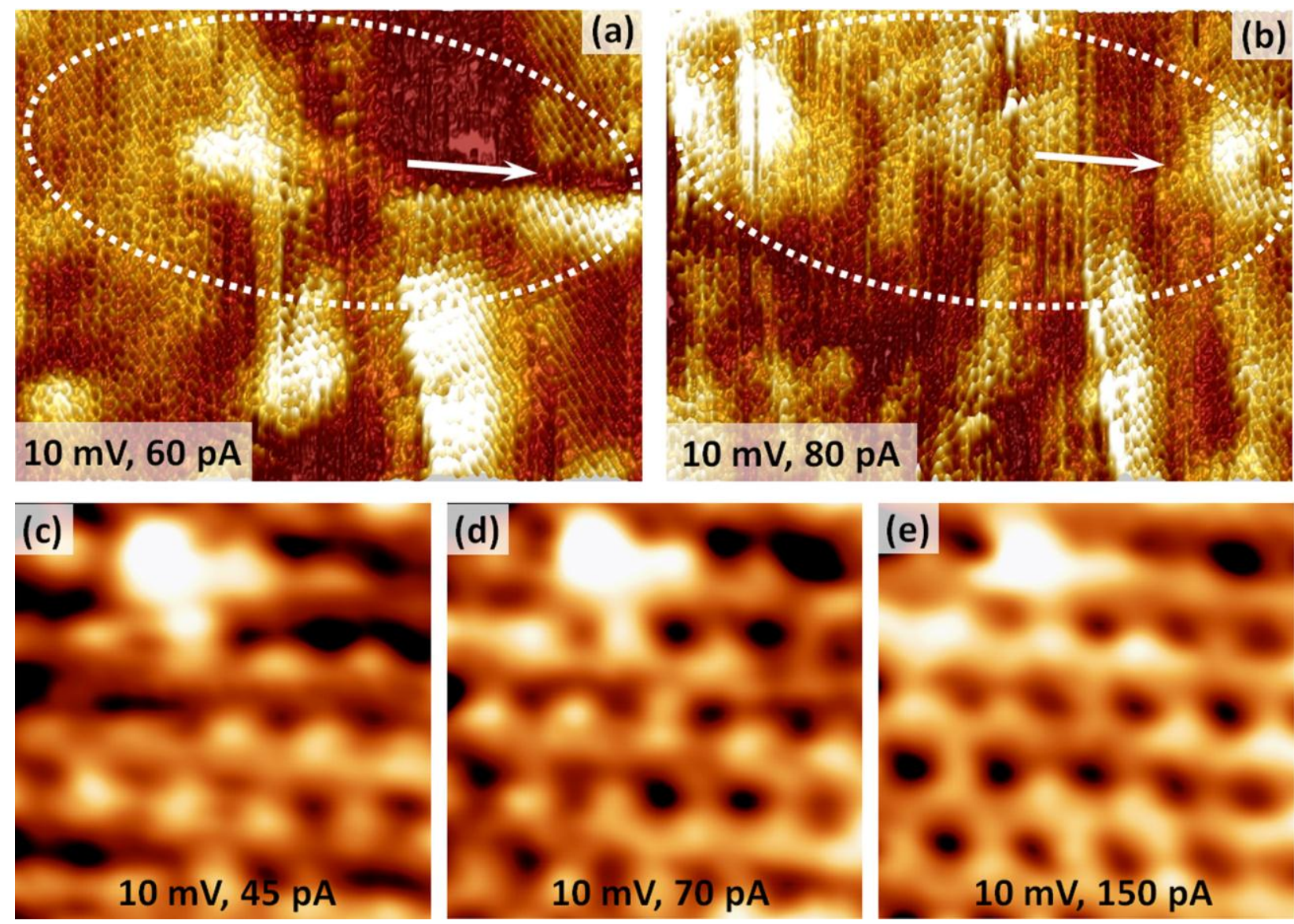

Figure 6: $(a, b)$ Consecutive atomically resolved $17 \times 13 \mathrm{~nm}^{2}$ STM images of the same surface region measured at the same bias voltage and different tunneling currents. The ovals in panels (a) and (b) indicate the surface area where the most substantial change in the surface layer roughness occurs. The arrows highlight a surface region appearing as a domain boundary in (a) and bent graphene layer in (b) at different tip-surface distances. The tunneling parameters are indicated on each particular frame. (c-e) Sequence of gap resistance-dependent STM images of the same surface region of graphene/ $\mathrm{SiC}(001)$ demonstrating the contrast reversal (from hexagonal to honeycomb pattern) with increasing tunneling current (decreasing tip-sample distance). The same defect is seen in the top left corners of the $12 \times 12 \AA^{2}$ images.

hexagonal (Fig. 5a) and honeycomb (Fig. 1d) symmetry can be resolved on the same surface areas depending on the tunneling parameters. This is illustrated by the gap-resistance dependence shown in Figs. 6c-e. The contrast inversion from honeycomb to hexagonal was reversibly and reproducibly observed in different surface areas. This effect is related only to the change in tunneling parameters used for STM imaging, rather than changes in the overlayer thickness. The contrast inversion at a small tipsample separation can be related to multiplescattering effects in the tunneling gap [37], atomic relaxations or modification of the orbital structure of the interacting tip and surface atoms at small tunneling gaps $[38,39]$. This can lead to a situation where the tunneling current is mostly governed by the tip atoms in the second layer rather than by the apex atom closest to the surface. Therefore, at some specific distances a maximum in the tunneling current can be observed when the tip is located above the hollow sites, and not above the true atomic positions. Therefore, researchers should bear in mind that the presence of honeycomb or hexagonal contrast in STM images should not be considered as a definitive measure of the overlayer's thickness.
Images measured near the boundaries (Figs. 7 and 8) demonstrate additional electron density modulations caused by edge states and defects in the honeycomb lattice. These can induce apparent roughness and change the periodicity of atomic features in images of graphene nanodomains [40-45]. STM images of domains with widths below $10 \mathrm{~nm}$ (Figs. 7e-h and 8) reveal additional electronic features due to the presence of defects near the domain boundaries. The superposition of the $(1 \times 1)$ and $(\sqrt{3} \times \sqrt{3}) \mathrm{R} 30^{\circ}$ patterns usually produced very complicated STM images of small domains where honeycomb or hexagonal lattices could only be resolved in some domain regions. Figures 7a-d illustrate the appearance of the $(\sqrt{3} \times \sqrt{3}) \mathrm{R} 30^{\circ}$ superstructure (Fig. 7b) near the boundaries of a $\sim 15$ nm-wide ribbon. STM images measured in domain regions far from the boundaries exhibit the typical honeycomb atomic structure of the surface layer (Fig. $7 \mathrm{~d})$. At the same time a $(\sqrt{3} \times \sqrt{3}) \mathrm{R} 30^{\circ}$ modulation is resolved near the ribbon edge (Fig. 7b). The FFT pattern shown in Fig. $7 \mathrm{c}$ reveals two rotated hexagons related to the $(1 \times 1)$ lattice and $(\sqrt{ } 3 \times \sqrt{3}) \mathrm{R} 30^{\circ}$ superstructure, respectively. Figs. 7e-h and 8 show that $(\sqrt{3} \times \sqrt{3}) \mathrm{R} 30^{\circ}$ patterns dominate in STM images of smaller domains. Note that different $(\sqrt{3} \times \sqrt{3}) \mathrm{R} 30^{\circ}$ 

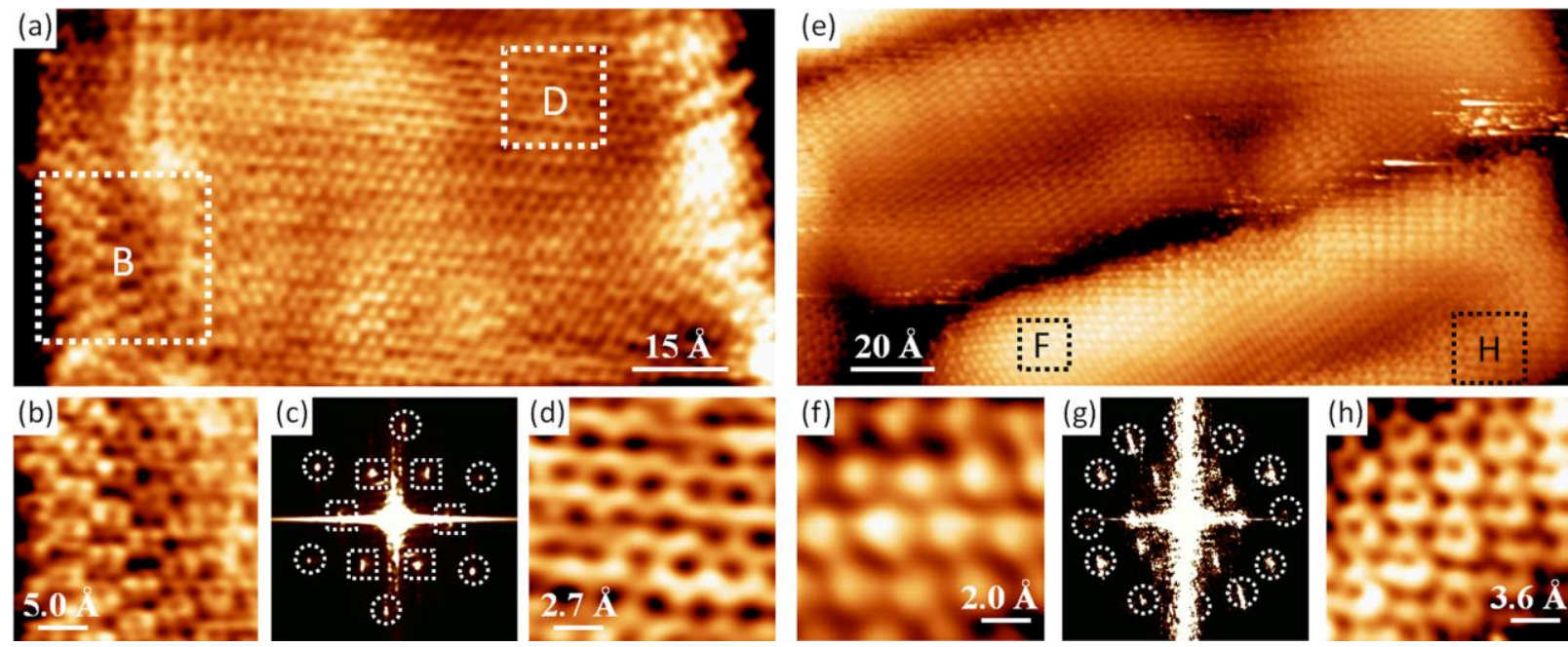

Figure 7: (a) STM image of a graphene nanoribbon demonstrating the appearance of $(\sqrt{3} \times \sqrt{3}) \mathrm{R} 30^{\circ}$ modulations near the ribbon edges. The dashed squares $\mathrm{B}$ and $\mathrm{D}$ indicate areas with $(\sqrt{3} \times \sqrt{3}) \mathrm{R} 30^{\circ}$ (b) and $(1 \times 1)$ honeycomb patterns $(\mathrm{d})$, respectively. (c) FFT pattern of the image in panel (a) showing two rotated hexagons related to the $(1 \times 1)$ (circles) and $(\sqrt{3} \times \sqrt{3}) \mathrm{R} 30^{\circ}$ (squares) periodicities. (e) STM image of two rotated domains. The dashed squares $\mathrm{F}$ and $\mathrm{H}$ indicate areas with the $(1 \times 1)$ hexagonal lattice (f) and $(\sqrt{3} \times \sqrt{3})$ R $30^{\circ}$ pattern (h), respectively. (g) FFT pattern of the image in panel (e) showing two systems of 12 non-equidistant spots related to the $(1 \times 1)$ (circles) and $(\sqrt{3} \times \sqrt{3}) \mathrm{R} 30^{\circ}$ periodicities. The images were measured at $U=10 \mathrm{mV}$ and $I=60 \mathrm{pA}$ (a) and $U=10 \mathrm{mV}$ and $I=70 \mathrm{pA}(\mathrm{e})$.
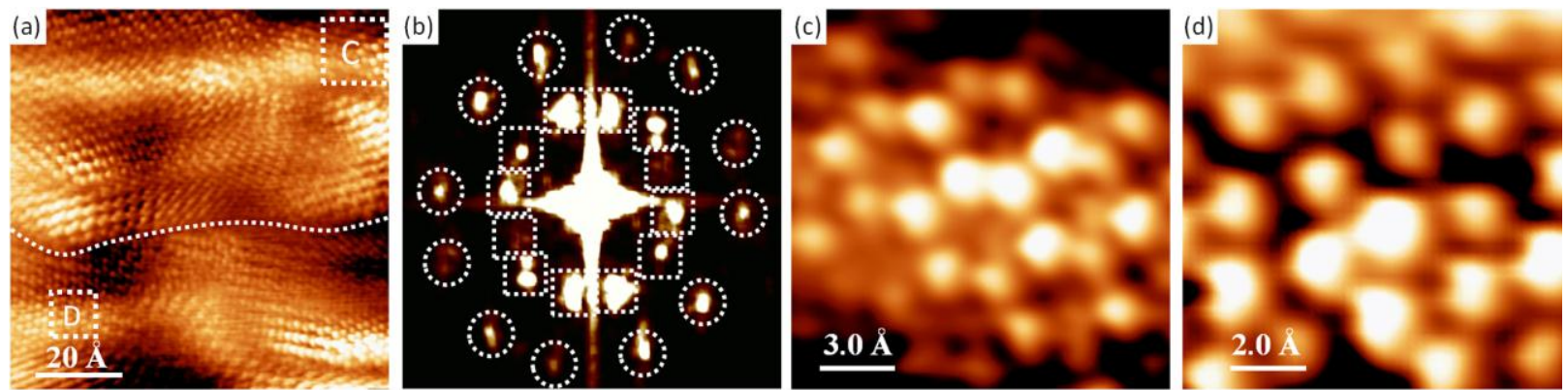

Figure 8: (a) STM image of rotated graphene domains on $\mathrm{SiC}(001)$ demonstrating the electronic interference effects. The image was taken at $U=-10 \mathrm{mV}$ and $I=80 \mathrm{pA}$. A white dashed line highlights the boundary between the domains. The dashed squares C and D indicate the surface areas shown in panels (c) and (d), respectively. (b) FFT pattern of the image in panel (a) showing two systems of 12 non-equidistant spots related to the $(1 \times 1)$ (circles) and $(\sqrt{3} \times \sqrt{3}) \mathrm{R} 30^{\circ}$ (squares) periodicity. (c,d) Zooms taken from the STM image in panel (a) demonstrating the $(\sqrt{3} \times \sqrt{3}) \mathrm{R} 30^{\circ}$ (c) and $(1 \times 1)$ patterns $(\mathrm{d})$.

patterns can be resolved even within the same domain (Figs. 7e and 8a), which can be related to different kinds of atomic defects at the boundaries and the diminishing amplitude of electron density modulations with increasing distance from the domain edges, as illustrated by Fig. 7a. The FFT patterns of the atomically resolved images of the rotated domains (Figs. $7 \mathrm{~g}$ and $8 \mathrm{~b}$ ) reveal two systems of twelve non-equidistant spots corresponding to $27^{\circ}$ rotated $(1 \times 1)$ and $(\sqrt{3} \times \sqrt{3}) \mathrm{R} 30^{\circ}$ patterns. The appearance of the $(\sqrt{3} \times \sqrt{3}) \mathrm{R} 30^{\circ}$ electronic interference patterns is typical of a quasi-freestanding graphene monolayer. Similar patterns were previously observed for graphene domains on graphite [40], $\mathrm{SiO}_{2}$ [44,45], and $\alpha-\mathrm{SiC}$ [41-43] which are considered as some of the highest quality graphene monolayers. The interference patterns resolved in our experiments on $\mathrm{SiC}(001)$ at small bias voltages demonstrate the close proximity of the $\pi$-like graphene states to the Fermi level [42] and indicate that these states in the topmost graphene layer are almost unperturbed by underlying layers. This is in agreement with our ARPES measurements, which reveal nearly ideal linear dispersions with the Dirac points close to the Fermi level for all four preferential orientations of the graphene domains [18].

\section{Conclusion}

STM and LEED data taken from different samples and surface regions prove the millimeter-scale continuity of the graphene layers on $\mathrm{SiC}(001)$, which consist of rotated nanodomains with four preferential orientations. Atomically resolved STM studies of different graphene domains show all the features typical of quasi-freestanding graphene (i.e., rippling, high flexibility of the topmost layer, interference patterns near defects and boundaries). The continuity of the domain network is not broken by the APD defects (Fig. 2c), which would otherwise be considered as a potential obstacle for the growth of uniform, continuous graphene coverage on cubic$\mathrm{SiC}$. However, the presence of domain boundaries can modify the electronic properties of graphene. Therefore, an increase in the domain size or greater control over the boundary directions can be 
considered as the next steps for improving graphene quality on cubic-SiC(001). It is suggested from the presented STM studies that the graphene domain size can be increased by minimizing the flashing time of the silicon-terminated $\mathrm{SiC}(001)-\mathrm{c}(4 \times 2)$ reconstruction, because continuous annealing could produce a lot of $\langle 110\rangle$-directed carbon chains [21], which can become grain boundaries after graphene synthesis (Figs. 1c and 2h). Subsequently, using vicinal $\mathrm{SiC}(001)$ substrates could achieve a preferential orientation of the carbon chains on the $\mathrm{SiC}(001)-\mathrm{c}(2 \times 2)$ reconstruction and improve the quality of the graphene/SiC(001) by aligning the graphene nanoribbons and grain boundaries along one of the two equivalent $\langle 110\rangle$ directions. Indeed, a reduction in the number of the rotated domain variants from four to two could be the reason for the uniform contrast observed in recent LEEM experiments conducted on the graphene/SiC(001) system synthesized on off-plane $\mathrm{Si}(001)$ wafers [16]. This result and the atomically resolved STM studies presented herein show it may be possible to synthesise a millimeter-scale graphene nanoribbon network with one preferential domain boundary direction on low-cost, off-plane $\mathrm{SiC}(001) / \mathrm{Si}(001)$ wafers.

\section{Acknowledgments}

This work was supported by the Russian Academy of Sciences, RFBR grants № 11-02-01253, 14-02-00949, 11-02-01256 and 14-02-01234, by a Marie Curie IIF grant within the $7^{\text {th }}$ European Community Framework Programme, Science Foundation Ireland Principal Investigator grant № 12/IA/1264, and by SPP 1459 of the Deutsche Forschungsgemeinschaft.

\section{References}

[1] Wallace P 1947 Phys. Rev. 71 622-634

[2] Novoselov K S, Geim A K, Morozov S V, Jiang D, Zhang Y, Dubonos S V, Grigorieva I V, Firsov A A 2004 Science 306 666-669

[3] Novoselov K S, Geim A K, Morozov S V, Jiang D, Katsnelson M I, Grigorieva I V, Dubonos S V, Firsov A A 2005 Nature 438 197-200

[4] Berger C, Zhimin Song, Tianbo Li, Xuebin Li, Ogbazghi A Y, Rui Feng, Zhenting Dai, Marchenkov A N, Conrad E H, First P N, de Heer W A $2004 J$. Phys. Chem. B 108 19912-19916

[5] Berger C, Zhimin Song, Xuebin Li, Xiaosong Wu, Brown N, Naud C, Mayou D, Li T, Hass J, Marchenkov A N, Conrad E H, First P N, de Heer W A 2006 Science 312 1191-1196

[6] Emtsev K V, Bostwick A, Horn K, Jobst J, Kellogg G L, Ley L, McChesney J, Ohta T, Reshanov S A, Rohr J, Rotenberg E, Schmid A K, Waldmann D, Weber H B, Seyller T 2009 Nat. Mater. 8 203-207

[7] Rutter G M, Guisinger N P, Crain J N, Jarvis J A, Stiles M D, Li T, First P N, Stroscio J A 2007 Phys. Rev. B 76235416
[8] Sprinkle M, Siegel D, Hu Y, Hicks J, Tejeda A, Taleb-Ibrahimi A, Le Fevre P, Bertran F, Vizzini S, Enriquez H, Chiang S, Soukiassian P, Berger C, de Heer W A, Lanzara A, Conrad E H 2009 Phys. Rev. Lett. 103226803

[9] Hass J, Varchon F, Millan-Otoya J E, Sprinkle M, Sharma N, de Heer W A, Berger C, First P N, Magaud L, Conrad E H 2008 Phys. Rev. Lett. 100 125504

[10] Wang Q, Zhang W, Wang L, He K, Ma X, Xue Q 2013 J. Phys.: Condens. Matter 25095002

[11] Hupalo M, Conrad E H, Tringides M C. 2009 Phys. Rev. B 80 041401(R)

[12] Huang H, Chen W, Chen S, Wee A T S 2008 ACS Nano 2 2513-2518

[13] Aristov V Yu, Urbanik G, Kummer K, Vyalikh D V, Molodtsova O V, Preobrajenski A B, Zakharov A A, Hess C, Hänke T, Büchner B, Vobornik I, Fujii J, Panaccione G, Ossipyan Yu A, Knupfer M 2010 Nano Letters 10 992-995

[14] Ouerghi A, Ridene M, Balan A, Belkhou R, Barbier A, Gogneau N, Portail M, Michon A, Latil S, Jegou P, Shukla A 2011 Phys. Rev. B 83205429

[15] Gogneau N, Balan A, Ridene M, Shukla A, Ouerghi A 2012 Surf. Sci. 606 217-220

[16] Ouerghi A, Balan A, Castelli C, Picher M, Belkhou R, Eddrief M, Silly M G, Marangolo M, Shukla A, Sirotti F 2012 Appl. Phys. Lett. 101 021603

[17] Huang H, Wong S L, Tin C, Luo Z Q, Shen Z X, Chen W, Wee A T S 2011 J. Appl. Phys. 110014308

[18] Chaika A N, Molodtsova O V, Zakharov A A, Marchenko D, Sanchez-Barriga J, Varykhalov A, Shvets I V, Aristov V Yu 2013 Nano Res. 6 562-570 [19] Yazyev O V, Louie S G 2010 Nature Mater. 9 806-809

[20] Derycke V, Soukiassian P, Mayne A, Dujardin G 2000 Surf. Sci. 446 L101-L107

[21] Derycke V, Soukiassian P, Mayne A, Dujardin G, Gautier J 1998 Phys. Rev. Lett. 81 5868-5871

[22] Semond F, Soukiassian P, Mayne A, Dujardin G, Douillard L, Jaussaud C 1997 Phys. Rev. Lett. 77 2013-2016

[23] Aristov V Yu, Douillard L, Fauchoux O, Soukiassian P 1997 Phys. Rev. Lett. 79 3700-3703

[24] Douillard L, Fauchoux O, Aristov V, Soukiassian P 2000 Appl. Surf. Sci. 166 220-223

[25] Soukiassian P, Semond F, Douillard L, Mayne A, Dujardin G, Pizzagalli L, Joachim C 1997 Phys. Rev. Lett. 78 907-910

[26] Douillard L, Aristov V Yu, Semond F, Soukiassian P 1998 Surf. Sci. 401 L395-L400

[27] Chaika A N, Nazin S S, Semenov V N, Bozhko S I, Lübben O, Krasnikov S A, Radican K, Shvets I V 2010 EPL 9246003

[28] Chaika A N, Nazin S S, Semenov V N, Orlova N N, Bozhko S I, Lübben O, Krasnikov S A, Radican K, Shvets I V 2013 Appl. Surf. Sci. 267 219-223

[29] Horcas I, Fernandez R, Gomez-Rodriguez J M, Colchero J, Gomez-Herrero J, Baro A M 2007 Rev. Sci. Instrum. 78013705

[30] Portail M, Michon A, Vezian S, Lefebvre D, Chenot S, Roudon E, Zielinski M, Chassagne T. 
Tiberj A, Camassel J, Cordier Y 2012 Journal of Crystal Growth 349 27-35

[31] Hass J, de Heer W A, Conrad E H 2008 J. Phys. Condens. Matter 20323202

[32] Meyer J C, Geim A K, Katsnelson M I, Novoselov K S, Booth T J, Roth S 2007 Nature 446 60-63

[33] Fasolino A, Los J H, Katsnelson M I 2007 Nature Mater. 6 858-861

[34] Klimov N N, Jung S, Zhu S, Li T, Wright C A, Solares S D, Newell D B, Zhitenev N B, Stroscio J A 2012 Science 336 1557-1561

[35] Zan R, Muryn C, Bangert U, Mattocks P, Wincott P, Vaughan D, Li X, Colombo L, Ruoff R S, Hamilton B, Novoselov K S 2012 Nanoscale 4 30653068

[36] Mashoff T, Pratzer M, Geringer V, Echtermeyer T J, Lemme M C, Liebmann M, Morgenstern M 2010 Nano Letters 10 461-465

[37] Ondracek M, Pou P, Rozsival V, Gonzalez S, Jelinek P, Perez R 2011 Phys. Rev. Lett. 106176101

[38] Chaika A N, Nazin S S, Semenov V N, Bozhko S I, Lübben O, Krasnikov S A, Radican K and Shvets I V 2010 EPL 9246003

[39] Grushko V I, Lubben O, Chaika A N, Novikov N, Mitskevich E, Chepugov A, Lysenko O, Murphy B E, Krasnikov S A, Shvets I V 2014 Nanotechnology 25025706

[40] Tapaszto L, Dobrik G, Lambin P, Biro L P 2008 Nature Nanotechnology 3 397-401

[41] Rutter G M, Crain J N, Guisinger N P, Li T, First P N, Stroscio J A 2007 Science 317 219-222

[42] Mallet P, Varchon F, Naud C, Magaud L, Berger C, Veuillen J-Y 2007 Phys. Rev. B 76 041403(R)

[43] Cockayne E, Rutter G M, Guisinger N P, Crain J N, First P N, Stroscio J A 2011 Phys. Rev. B 83 195425

[44] Deshpande A, Bao W, Miao F, Lau C N, LeRoy B J 2009 Phys. Rev. B 79205411

[45] Deshpande A, LeRoy B J 2012 Physica E 44 743-759 